\title{
Impacto do Diabetes Melito na Eficácia e na Segurança dos Stents Farmacológicos: Registro com Até Cinco Anos de Seguimento
}

\author{
Luis Felipe Smidt' ${ }^{1}$ Rodrigo Bodanese', Vitor Gomes ${ }^{1}$, Ricardo Lasevitch ${ }^{1}$, Christiano Barcellos ${ }^{1}$, \\ Denise Oliveira ${ }^{1}$, Marina Morais ${ }^{1}$, Patrícia Hickmann ${ }^{1}$, Ana Krepsky ${ }^{1}$, Marcelo Arndt ${ }^{1}$, \\ Carisi Polanczyk², Luiz Carlos Bodanese', Paulo Caramori'
}

\begin{abstract}
RESUMO
Introdução: Diabéticos possuem risco aumentado para ocorrência de eventos cardiovasculares. Estudos revelam que os stents farmacológicos reduzem a reestenose e a revascularização da lesão-alvo em pacientes diabéticos e não-diabéticos. Entretanto, poucos estudos avaliam a segurança e a eficácia dos stents farmacológicos em diabéticos no longo prazo. O objetivo deste trabalho foi avaliar os desfechos clínicos tardios nessa população de alto risco. Método: No período de maio de 2002 a abril de 2007, todos os pacientes submetidos a intervenção coronária com stents farmacológicos em dois hospitais foram incluídos no estudo e acompanhados por até cinco anos. Resultados: No total, 611 pacientes com média de idade de $63,5 \pm 11,2$ anos foram incluídos neste registro. Os diabéticos $(n=204,33,4 \%)$ apresentaram menor proporção de pacientes do sexo masculino, maior prevalência de hipertensão arterial e insuficiência renal crônica, e menor diâmetro de referência do vaso em comparação aos não-diabéticos. Durante o seguimento, os diabéticos mostraram maior probabilidade de eventos cardíacos adversos combinados $(19,7 \%$ vs. $13,4 \%$; $P=0,04)$, em decorrência de óbito $(7,4 \%$ vs. $2,3 \% ; P=0,003)$, infarto agudo do miocárdio $(5,9 \%$ vs. $3,1 \% ; \mathrm{P}=0,10)$ e trombose do stent definitiva/provável $(3,9 \%$ vs. $1,3 \% ; P=0,04)$. A revascularização do vaso-alvo e a revascularização da lesão-alvo, entretanto, não diferiram entre os grupos $(10,9 \%$ vs. $9,8 \% ; P=0,68$ e $3,4 \%$ vs. $5,1 \% ; P=0,35$, respectivamente). A presença de diabetes melito foi preditora independente de óbito [odds ratio (OR) 2,42; intervalo de confiança de 95\% (IC 95\%) 1,02-5,78; P = 0,05], mas não de trombose de stent (OR 2,41; IC 95\% 0,76-7,61; P = 0,13), em nossos pacientes. Conclusões: Os resultados deste estudo demonstraram que, em pacientes submetidos a implante
\end{abstract}

\footnotetext{
1 Hospital São Lucas da Pontifícia Universidade Católica do Rio Grande do Sul - Porto Alegre, RS, Brasil.

2 Universidade Federal do Rio Grande do Sul (UFRGS) - Porto Alegre, RS, Brasil.

Correspondência: Paulo Caramori. Serviço de Hemodinâmica Hospital São Lucas - Av. Ipiranga, 6.690 - Jardim Botânico - Porto Alegre, RS, Brasil - CEP 90610-000

E-mail: caramori.p@plugin.com.br

Recebido em: 10/9/2009 • Aceito em: 1\%/12/2009
}

\section{ABSTRACT}

\section{Impact of Diabetes Mellitus in the Efficacy and Safety of Drug-Eluting Stents: A Five-Year Follow-up Registry}

Background: Diabetic patients are at high risk of recurrent coronary events. Drug eluting stents (DES) reduce restenosis and target lesion revascularization in both diabetic and nondiabetic patients. However, there are limited data on the long-term safety and efficacy of DES in diabetic patients. The objective of this study was to evaluate the long-term clinical outcome in this high risk population. Method: From May 2002 to April 2007, all patients undergoing coronary intervention with DES in two hospitals were included in the study and were followed-up for up to 5 years. Results: A total of 611 patients with mean age of $63.5 \pm 11.2$ years were included in the registry. Diabetic patients $(n=204$, $33.4 \%$ ) included a smaller proportion of males, greater prevalence of hypertension, chronic renal failure, and smaller coronary reference diameter when compared to non-diabetic patients. During the follow-up, diabetics had a higher probability of combined adverse cardiac events $(19.7 \%$ vs. $13.4 \%$; $P=0.04)$, as a result of death $(7.4 \%$ vs $2.3 \% ; P=0.003)$, acute myocardial infarction $(5.9 \%$ vs. $3.1 \% ; \mathrm{P}=0.10)$ and definitive/probable stent thrombosis $(3.9 \%$ vs. $1.3 \% ; P=0.04)$. Target vessel and target lesion revascularization, however, were not different between the groups $(10.9 \%$ vs. $9.8 \% ; P=0.68$ and $3.4 \%$ vs. $5.1 \%$; $\mathrm{P}=0.35$, respectively). The presence of diabetes mellitus was an independent predictor of death [odds ratio (OR) $2.41 ; 95 \%$ confidence interval $(95 \% \mathrm{Cl}) 1.02-5.78 ; \mathrm{P}=0.05)$ but not of stent thrombosis (OR 2.41;95\% Cl 0.76-7.61; $P=0.13)$ in our patients. Conclusions: The outcomes of this study show that in patients undergoing DES implantation, 
Smidt LF, et al. Impacto do Diabetes Melito na Eficácia e na Segurança dos Stents Farmacológicos: Registro com Até Cinco Anos de Seguimento. Rev Bras Cardiol Invas. 2009;17(4):505-11.

de stents farmacológicos, a presença de diabetes esteve associada a maiores taxas de mortalidade, infarto agudo do miocárdio e trombose do stent, comparativamente aos não-diabéticos. A presença de taxas similares de revascularização da lesão-alvo em diabéticos e não-diabéticos é promissora e sugere a eficácia da revascularização percutânea com implante de stents farmacológicos nessa população.

DESCRITORES: Diabetes mellitus. Stents farmacológicos. Reestenose coronária. Seguimentos.

A doença arterial coronária tem aumentado sua prevalência em todo o mundo ${ }^{1}$. Associadamente, a prevalência do diabetes melito vem crescendo de maneira epidêmica ${ }^{2}$. Os diabéticos representam hoje quase $35 \%$ dos pacientes com aterotrombose ${ }^{3}$ e $25 \%$ dos pacientes submetidos a revascularização coronária ${ }^{4}$. Aproximadamente $75 \%$ das mortes em diabéticos ocorrem como consequência direta da doença arterial coronária ${ }^{5}$.

Pacientes diabéticos têm alto risco para a ocorrência de eventos cardiovasculares por uma série de fatores, dentre eles maior incidência de doença em múltiplos vasos e pior função ventricular. As características angiográficas e ultrassonográficas mais comuns são: maior carga de placa aterosclerótica, remodelamento vascular negativo, lesões mais difusas, menor diâmetro de referência do vaso e menor formação de colaterais $^{6-8}$. Além disso, observa-se maior atividade inflamatória ${ }^{9}$ e trombogênica e associação frequente com outras comorbidades ${ }^{10}$.

Os stents farmacológicos reduzem as taxas de revascularização da lesão-alvo e do vaso-alvo em pacientes diabéticos e não-diabéticos, quando comparados aos stents convencionais ${ }^{11,12}$. No entanto, os diabéticos apresentam resposta neointimal exacerba$\mathrm{da}^{13}$, o que aumenta o risco de reestenose em relação aos não-diabéticos. Além disso, o diabetes melito também está associado a trombose do stent, tanto aguda como tardia ${ }^{14-16}$.

Poucos estudos têm avaliado a segurança e a eficácia dos stents farmacológicos em diabéticos no longo prazo, sobretudo em nosso meio. Assim, este estudo teve como objetivo avaliar os desfechos clínicos tardios nos pacientes dessa população de alto risco tratados por stents farmacológicos.

\section{MÉTODO}

\section{População do estudo}

A amostra deste estudo compreendeu todos os pacientes submetidos a intervenção coronária percutânea com o uso de stents farmacológicos $\left(\operatorname{Costar}^{\mathrm{TM}}{ }^{\text {, Cypher }}{ }^{\mathrm{TM}}\right.$, the presence of diabetes was associated to higher mortality rates, acute myocardial infarction and stent thrombosis when compared to non-diabetics. The finding of similar target lesion revascularization rates in diabetic and nondiabetic patients is promising and suggests the efficacy of percutaneous revascularization with DES implantation in this population.

DESCRIPTORS: Diabetes mellitus. Drug-eluting stents. Coronary restenosis. Follow-up studies.

Endeavor $^{\mathrm{TM}}$, Infinnium ${ }^{\mathrm{TM}}$, Janus ${ }^{\mathrm{TM}}$, Supralimus ${ }^{\mathrm{TM}}$ e Taxus $^{\text {TM }}$ ), no período de maio de 2002 a abril de 2007, no Hospital São Lucas e no Hospital Mãe de Deus, em Porto Alegre (RS), incluindo portadores de síndrome coronária aguda com e sem supradesnivelamento do segmento ST. Para pacientes com mais de uma intervenção durante o período de acompanhamento deste estudo, somente o primeiro procedimento foi considerado para análise. A presença de diabetes melito foi confirmada pela revisão cruzada de prontuários realizada por dois médicos experientes. Do total de 611 pacientes, 204 (33,4\%) eram diabéticos.

\section{Definições e seguimento clínico}

A extensão da doença arterial coronária foi definida pelo número de coronárias com estenose luminal $\geq 70 \%$. Revascularização do vaso-alvo foi definida como a necessidade de revascularização percutânea ou cirúrgica no vaso previamente tratado. Revascularização da lesão-alvo foi definida como a necessidade de nova revascularização percutânea ou cirúrgica na lesão previamente tratada. A definição de trombose definitiva e provável do stent foi baseada na classificação do Academic Research Consortium (ARC) ${ }^{17}$.

O seguimento clínico foi realizado por meio de revisão dos prontuários médicos, contato com o médico assistente ou entrevistas telefônicas. Avaliou-se a ocorrência de eventos cardiovasculares adversos maiores agrupados (morte, infarto agudo do miocárdio, revascularização do vaso-alvo ou trombose do stent) e isolados.

\section{Angiografia coronária quantitativa}

A análise angiográfica quantitativa foi realizada off-line (CardioNow Websend DICOM Study Sharing Software, HeartLab, Inc., Westerly, Estados Unidos). O diâmetro de referência, o diâmetro luminal mínimo e o diâmetro da estenose foram medidos na projeção que apresentasse o maior grau de obstrução (worst view), antes e depois da intervenção.

\section{Análise estatística}

A análise estatística foi realizada utilizando o software SPPS 12.0 (SPSS Inc., Chicago, Estados Uni- 
Smidt LF, et al. Impacto do Diabetes Melito na Eficácia e na Segurança dos Stents Farmacológicos: Registro com Até Cinco Anos de Seguimento. Rev Bras Cardiol Invas. 2009;17(4):505-11.

dos). As variáveis categóricas foram apresentadas como frequências e porcentuais e comparadas pelo teste de qui-quadrado ou teste exato de Fisher, quando apropriado. As variáveis contínuas foram apresentadas como média \pm desvio padrão e comparadas pelo teste $t$ de Student ou pelo teste de Mann-Whitney. Curvas de Kaplan-Meier e teste de log-rank foram realizados para a análise dos desfechos clínicos. A regressão multivariada de Cox foi realizada com as variáveis que alcançaram $\mathrm{P}<0,10$ na análise univariada. $\mathrm{O}$ valor de $\mathrm{P}<0,05$ foi considerado estatisticamente significante.

\section{RESULTADOS}

De um total de 611 pacientes tratados com stents farmacológicos em nosso serviço, 595 (97,4\%) tinham informações a respeito da presença ou não de diabetes melito, sendo incluídos em nosso estudo. A Tabela 1 mostra as características clínicas e as indicações para intervenção nos pacientes diabéticos e não-diabéticos. Os diabéticos, dos quais $36,5 \%$ estavam em uso de insulina, apresentaram menor porcentual de pacientes do sexo masculino $(58,3 \%$ vs. $66 \%$; $P=0,07)$ e idade semelhante $(63,9 \pm 10,9$ anos vs. $63 \pm 12,1$ anos; $\mathrm{P}=0,39)$ quando comparados aos não-diabéticos. Apresentaram, ainda, maior prevalência de hipertensão arterial ( $83,7 \%$ vs. $75,6 \% ; P=0,02)$ e de insuficiência renal crônica $(6,9 \%$ vs. 0,8\%; P < 0,001). Angina estável foi o quadro clínico predominante préintervenção percutânea em ambos os grupos (59,9\% vs. $60,4 \%$ )
Em relação às características angiográficas e do procedimento (Tabela 2), mais da metade dos pacientes tinha acometimento multiarterial $(62,2 \%$ vs. $56,5 \%$; $\mathrm{P}=0,28)$, a artéria descendente anterior foi o vaso mais frequentemente tratado (55,9\% vs. 49,7\%) e predominaram as lesões complexas B2/C em ambos os grupos. O diâmetro de referência do vaso foi significantemente menor nos diabéticos $(2,81 \pm 0,45 \mathrm{~mm}$ vs. $2,90 \pm 0,46 \mathrm{~mm} ; \mathrm{P}=0,05)$, mas a extensão da lesão foi semelhante nos grupos de diabéticos e não-diabéticos $(16,36 \pm 8,56 \mathrm{~mm}$ vs. 15,53 $\pm 8,38 \mathrm{~mm}$, respectivamente; $P=0,27)$. O sucesso angiográfico foi alto e sem diferença entre os grupos $(97,4 \%$ vs. $98,6 \%$; $P=0,49)$.

$\mathrm{Na}$ fase hospitalar, os pacientes diabéticos evoluíram com maior porcentual de óbitos $(2,5 \%$ vs. 0 ; $\mathrm{P}=0,08)$, mas igual incidência de infarto agudo do miocárdio $(1,5 \%$ vs. $1,3 \% ; P>0,99)$ e sucesso clínico semelhante (96\% vs. $97,7 \% ; P=0,27)$.

Na evolução tardia, os diabéticos mostraram maior probabilidade de eventos cardiovasculares maiores $(19,7 \%$ vs. $13,4 \%$; $P=0,04)$, em decorrência de óbito (7,4\% vs. $2,3 \% ; P=0,03)$, infarto agudo do miocárdio $(5,9 \%$ vs. $3,1 \% ; P=0,10)$ e trombose do stent definitiva/provável $(3,9 \%$ vs. $1,3 \% ; P=0,04)$. A revascularização do vaso-alvo e a revascularização da lesãoalvo, entretanto, não diferiram entre os grupos $(10,9 \%$ vs. $9,8 \%$; $P=0,68$ e $3,4 \%$ vs. $5,1 \% ; P=0,35$, respectivamente) (Tabela 3).

TABELA 1

Características basais da população de pacientes

\begin{tabular}{|c|c|c|c|}
\hline & $\begin{array}{l}\text { Diabéticos } \\
n=204\end{array}$ & $\begin{array}{c}\text { Não-diabéticos } \\
\qquad \mathbf{n}=391\end{array}$ & $\mathbf{P}$ \\
\hline Sexo masculino, \% & 58,3 & 66 & 0,07 \\
\hline Idade, anos* & $63,9 \pm 10,9$ & $63 \pm 12,1$ & 0,39 \\
\hline Hipertensão arterial, \% & 83,7 & 75,6 & 0,02 \\
\hline História familiar de DAC, \% & 47,9 & 51,9 & 0,36 \\
\hline Tabagismo ativo, \% & 7,8 & 9 & 0,68 \\
\hline Dislipidemia, \% & 78,8 & 76 & 0,44 \\
\hline Insuficiência renal crônica, \% & 6,9 & 0,8 & $<0,001$ \\
\hline Creatinina sérica, mg/dl* & $1,29 \pm 0,96$ & $1,16 \pm 0,78$ & 0,09 \\
\hline ICP prévia, \% & 33,2 & 26,6 & 0,09 \\
\hline CRM prévia, \% & 10,4 & 11,5 & 0,69 \\
\hline Quadro clínico, \% & & & 0,24 \\
\hline Angina estável & 59,9 & 60,4 & \\
\hline Angina instável & 28,2 & 23,2 & \\
\hline IAM & 7,9 & 12,8 & \\
\hline IAM recente & 3,9 & 3,6 & \\
\hline
\end{tabular}

* Valores expressos em média \pm desvio padrão.

AAS = ácido acetilsalicílico; CRM = cirurgia de revascularização do miocárdio; DAC = doença arterial coronária; IAM = infarto agudo do miocárdio; ICP = intervenção coronária percutânea; $\mathrm{n}=$ número de pacientes. 
TABELA 2

Características angiográficas e do procedimento

\begin{tabular}{|c|c|c|c|}
\hline & $\begin{array}{l}\text { Diabéticos } \\
n=204\end{array}$ & $\begin{array}{c}\text { Não-diabéticos } \\
n=391\end{array}$ & $\mathbf{P}$ \\
\hline Vaso-alvo, \% & & & 0,10 \\
\hline Descendente anterior & 55,9 & 49,7 & \\
\hline Circunflexa & 11,9 & 10,6 & \\
\hline Coronária direita & 23,3 & 23,2 & \\
\hline Tronco de coronária esquerda & 0,5 & 2,8 & \\
\hline Doença multivascular ( $\geq 2$ vasos), \% & 62,2 & 56,5 & 0,28 \\
\hline Tipo de lesão (ACC/AHA), \% & & & 0,60 \\
\hline A & 0,5 & 0,6 & \\
\hline B1 & 2,1 & 3,1 & \\
\hline B2 & 45,3 & 50 & \\
\hline $\mathrm{C}$ & 52,1 & 46,4 & \\
\hline Reestenose intrastent, \% & 12,4 & 8,1 & 0,10 \\
\hline Oclusão total crônica, \% & 6,2 & 6,7 & 0,83 \\
\hline Lesão ostial, \% & 14,2 & 8,9 & 0,07 \\
\hline \multicolumn{4}{|l|}{ ACQ pré-procedimento } \\
\hline Diâmetro de referência proximal, mm* & $2,81 \pm 0,45$ & $2,90 \pm 0,46$ & 0,05 \\
\hline Diâmetro luminal mínimo, mm* & $0,96 \pm 0,50$ & $0,96 \pm 0,83$ & 0,99 \\
\hline Extensão da lesão, mm* & $16,36 \pm 8,56$ & $15,53 \pm 8,38$ & 0,27 \\
\hline \multicolumn{4}{|l|}{ ACQ pós-procedimento } \\
\hline Diâmetro do stent, mm* & $2,93 \pm 0,35$ & $2,93 \pm 0,34$ & 0,98 \\
\hline Extensão do stent, mm* & $20,41 \pm 5,60$ & $19,74 \pm 6,20$ & 0,20 \\
\hline Pressão máxima de implante do stent, mm* & $14,98 \pm 3,06$ & $14,80 \pm 2,61$ & 0,49 \\
\hline Diâmetro de referência, mm* & $2,86 \pm 0,45$ & $2,91 \pm 0,46$ & 0,30 \\
\hline Diâmetro luminal mínimo, mm* & $2,79 \pm 0,50$ & $2,83 \pm 0,47$ & 0,29 \\
\hline Ganho agudo, mm* & $1,82 \pm 0,66$ & $1,91 \pm 0,63$ & 0,14 \\
\hline Sucesso angiográfico, \% & 97,4 & 98,6 & 0,49 \\
\hline
\end{tabular}

* Valores expressos em média \pm desvio padrão.

$\mathrm{ACC} / \mathrm{AHA}=$ American College of Cardiology/American Heart Association; $\mathrm{ACQ}=$ angiografia coronária quantitativa; $\mathrm{n}=$ número de pacientes.

TABELA 3

Principais desfechos clínicos e fármacos em uso no acompanhamento de cinco anos

\begin{tabular}{lccc}
\hline & $\begin{array}{c}\text { Diabéticos } \\
\mathbf{n = 2 0 4}\end{array}$ & $\begin{array}{c}\text { Não-diabéticos } \\
\mathbf{n = 3 9 1}\end{array}$ & $\mathbf{P}$ \\
\hline ECVM, \% & 19,7 & 13,4 & 0,04 \\
Morte, \% & 7,4 & 2,3 & 0,03 \\
IAM, \% & 5,9 & 3,1 & 0,10 \\
Revascularização do vaso-alvo, \% & 10,9 & 9,8 & 0,68 \\
Revascularização da lesão-alvo, \% & 3,4 & 5,1 & 0,35 \\
Trombose do stent (definitiva/provável), \% & 3,9 & 1,3 & 0,04 \\
Fármacos em uso, \% & & & 0,49 \\
AAS & 82,4 & 84,6 & 0,92 \\
Tienopiridínicos & 52,1 & 51,7 & 0,42 \\
Estatinas & 85,3 & 87,5 & 0,52 \\
IECA & 42,2 & 39,4 & 0,17 \\
ARA 2 & 20,9 & 16,2 & 0,17 \\
Betabloqueadores & 55,6 & 61,6 & \\
\hline
\end{tabular}

AAS = ácido acetilsalicílico; ARA 2 = antagonistas dos receptores da angiotensina 2; ECVM = eventos cardiovasculares maiores; IAM = infarto agudo do miocárdio; IECA = inibidores da enzima de conversão do angiotensina; $\mathrm{n}=$ número de pacientes. 


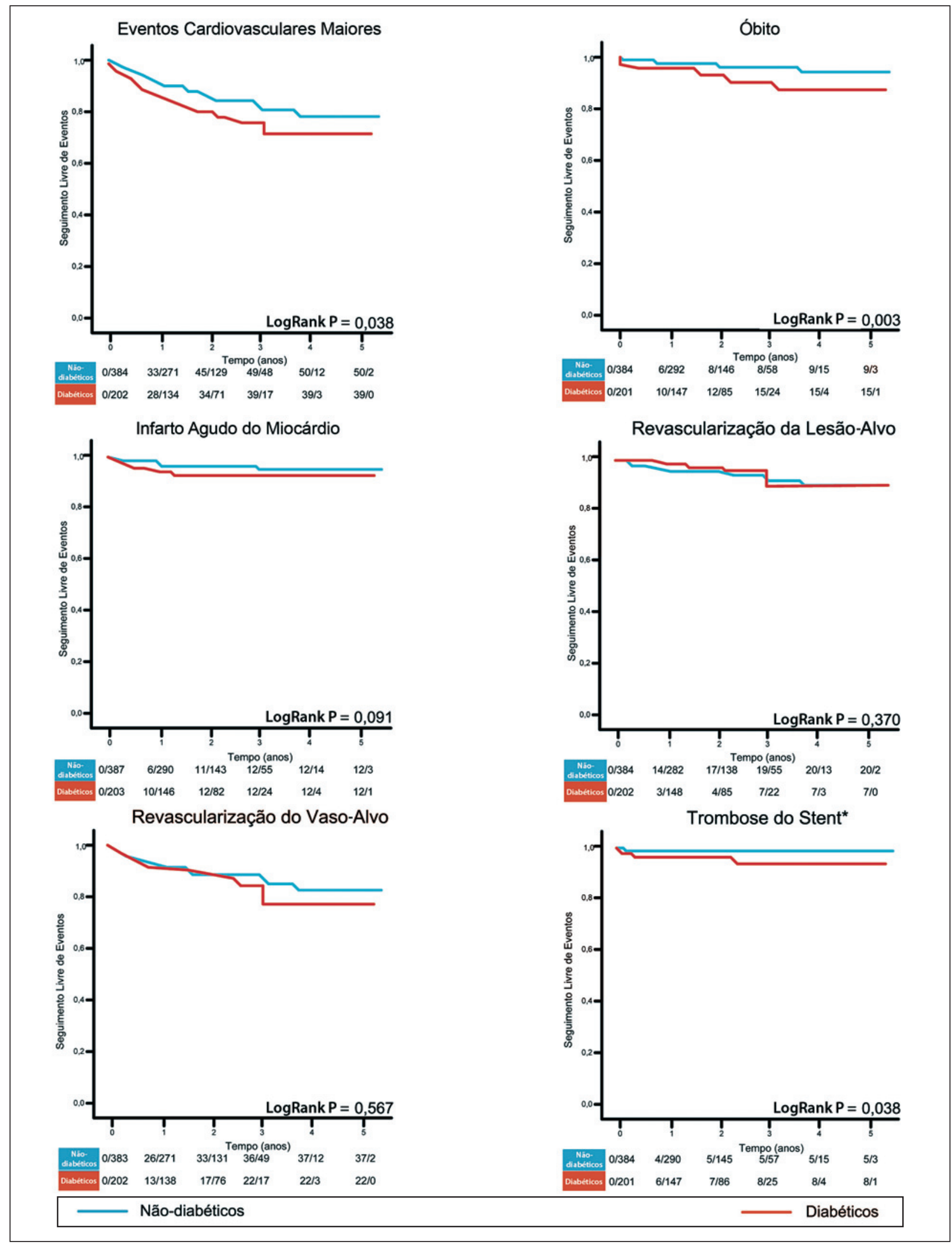

* Trombose definida/provável.

Figura 1 - Curvas de Kaplan-Meier com seguimento livre de eventos de até cinco anos em diabéticos e não-diabéticos. 
Smidt LF, et al. Impacto do Diabetes Melito na Eficácia e na Segurança dos Stents Farmacológicos: Registro com Até Cinco Anos de Seguimento. Rev Bras Cardiol Invas. 2009;17(4):505-11.

As curvas de Kaplan-Meier para eventos cardiovasculares maiores e óbito divergiram precocemente, mostrando maior probabilidade desses eventos para os pacientes diabéticos que receberam stents farmacológicos. Já as curvas de trombose do stent começaram a se afastar por volta do segundo ano, novamente mostrando maior probabilidade de trombose para os diabéticos e mantendo essa diferença até o fim do seguimento. Por fim, as curvas de revascularização da lesão-alvo mostraram-se superponíveis ao longo de todo o acompanhamento em ambos os grupos (Figura 1).

$\mathrm{Na}$ análise multivariada, a presença do diabetes melito foi preditora independente de óbito (OR 2,42; IC 95\% 1,02-5,78; P = 0,05), mas não de trombose de stent (OR 2,41; IC 95\% 0,76-7,61; $\mathrm{P}=0,13$ ) nesse grupo de pacientes.

\section{DISCUSSÃO}

Neste estudo pôde-se constatar que diabéticos, comparados a não-diabéticos, tratados com stents farmacológicos apresentam: 1) mortalidade hospitalar mais elevada; 2) taxas de mortalidade, infarto agudo do miocárdio e trombose do stent tardios significativamente maiores na análise univariada; e 3) iguais cifras de revascularização da lesão-alvo e de revascularização do vasoalvo tardias. Adicionalmente, o diabetes foi identificado como preditor independente de mortalidade tardia.

Estudos que avaliaram pacientes tratados com stents farmacológicos demonstram maior mortalidade em diabéticos, explicada em geral pela maior recorrência de eventos cardiovasculares ${ }^{14-16,18}$. O paciente diabético também cursa com maior número de comorbidades ${ }^{19}$ e as maiores cifras de hipertensão arterial e de insuficiência renal crônica encontradas neste estudo, associadas ao menor diâmetro do vaso tratado, que explicam, em parte, o maior risco de eventos cardiovasculares nesses pacientes.

Algumas publicações recentes, com maior número de pacientes, demonstraram o diabetes melito como preditor independente de trombose do stent ${ }^{14-16}$. Pacientes diabéticos possuem maior predisposição a trombose do stent, em decorrência da atividade inflamatória e trombogênica exacerbada, comparativamente aos não-diabéticos ${ }^{6-8}$. Caracteristicamente, esses pacientes apresentam resposta diminuída aos antiagregantes plaquetários, sustentação da terapia farmacológica antitrombogênica após implante de stents farmacológicos ${ }^{20,21}$. Neste estudo, o diabetes não foi preditor independente de trombose do stent, possivelmente pelo baixo número absoluto de eventos nesta amostra.

Estudos realizados com stent convencional demonstraram o diabetes melito como preditor de reestenose clínica e angiográfica após intervenções coronárias percutâneas ${ }^{22,23}$. Fatores relacionados à anatomia coronária concorrem para o maior risco de reestenose em pacientes diabéticos, como menor diâmetro de referência do vaso, menor diâmetro luminal do vaso pós-stent e uso de stents mais longos ${ }^{24,25}$. Os stents farmacológicos foram eficazes em reduzir a reestenose tanto clínica como angiográfica ${ }^{11,12}$. Este estudo demonstrou que, em seguimento de longo prazo, o diabetes melito não exerceu impacto significativo nas taxas de revascularização em pacientes após implante de stents farmacológicos. Baixos índices de revascularização do vaso-alvo e de revascularização da lesão-alvo já haviam sido demonstrados em meta-análise com o uso de stents com eluição de sirolimus e de stents com eluição de paclitaxel ${ }^{26}$. Risco semelhante de revascularização da lesão-alvo entre diabéticos e nãodiabéticos foi demonstrado em análise recente de um registro multicêntrico europeu ${ }^{27}$. Os resultados deste trabalho estão de acordo com esses estudos e demonstram que os stents farmacológicos podem anular o maior risco de reestenose dos diabéticos, igualando seus resultados aos dos não-diabéticos.

\section{LIMITAÇÕES}

Este estudo possui limitações inerentes a um registro. Avaliou-se a reestenose clínica, não havendo seguimento angiográfico ou realização de exames funcionais de rotina. O número de pacientes insulinodependentes foi pequeno, o que impediu a análise específica de seus desfechos. Por fim, a falta de um grupo controle com pacientes tratados com stents convencionais não permitiu a comparação com stents farmacológicos.

\section{CONCLUSÃO}

Neste registro, com seguimento de longo prazo de pacientes submetidos a implante de stents farmacológicos, a presença de diabetes melito esteve associada a maiores taxas de mortalidade, infarto agudo do miocárdio e trombose do stent, em relação aos nãodiabéticos. Apesar disso, taxas similares de revascularização da lesão-alvo em diabéticos e não-diabéticos é achado promissor e sugere a eficácia da revascularização percutânea com implante de stents farmacológicos nessa população.

\section{CONFLITO DE INTERESSES}

Os autores declararam inexistência de conflito de interesses relacionado a este manuscrito.

\section{REFERÊNCIAS BIBLIOGRÁFICAS}

1. Sanderson JE, Mayosi B, Yusuf S, Reddy S, Hu S, Chen Z, et al. Global burden of cardiovascular disease. Heart. 2007;93(10): 1175. Comment in: Heart. 2007;93(10):1176-83.

2. Smith SC Jr. Multiple risk factors for cardiovascular disease and diabetes mellitus. Am J Med. 2007;120(3 Suppl 1): S3-S11. Review.

3. Bhatt DL, Steg PG, Ohman EM, Hirsch AT, Ikeda Y, Mas JL, Goto S, Liau CS, Richard AJ, Röther J, Wilson PW; REACH Registry Investigators. International prevalence, recognition, and treatment of cardiovascular risk factors in outpatients with atherothrombosis. JAMA. 2006;295(2):180-9. 
Smidt LF, et al. Impacto do Diabetes Melito na Eficácia e na Segurança dos Stents Farmacológicos: Registro com Até Cinco Anos de Seguimento. Rev Bras Cardiol Invas. 2009;17(4):505-11.

4. Flaherty JD, Davidson CJ. Diabetes and coronary revascularization. JAMA. 2005;293(12):1501-8.

5. Laakso M. Hyperglicemia and cardiovascular disease in type 2 diabetes. Diabetes. 1999;48(5):937-42.

6. Jensen LO, Maeng M, Thayssen P, Christiansen EH, Hansen KN, Galloe A, et al. Neointimal hyperplasia after sirolimuseluting and paclitaxel-eluting stent implantation in diabetic patients: The Randomized Diabetes and Drug-Eluting Stent (diabeDES) Intravascular Ultrasound Trial. Eur Heart J. 2008; 29(22):2733-41

7. Jensen LO, Maeng M, Mintz GS, Christiansen EH, Hansen $\mathrm{KN}$, Galloe A, et al. Intravascular ultrasound assessment of expansion of the sirolimus-eluting (cypher select) and paclitaxeleluting (Taxus Express-2) stent in patients with diabetes mellitus. Am J Cardiol. 2008;102(1):19-26.

8. Abaci A, Oðuzhan A, Kahraman S, Eryol NK, Unal S, Arinç $\mathrm{H}$, et al. Effect of diabetes mellitus on formation of coronary collateral vessels. Circulation. 1999;99(17):2239-42.

9. Saito I, Folsom AR, Brancati FL, Duncan BB, Chambless LE. McGovern PG. Nontraditional risk factors for coronary heart disease incidence among persons with diabetes: the Atherosclerosis Risk in Communities (ARIC) Study. Ann Intern Med. 2000;133(2):81-91. Comment in: Ann Intern Med. 2000; 133(2):154-6.

10. Kip KE, Faxon DP, Detre KM, Yeh W, Kelsey SF, Currier JW. Coronary angioplasty in diabetic patients. The National Heart, Lung, and Blood Institute Percutaneous Transluminal Coronary Angioplasty Registry. Circulation. 1996;94(8):1818-25. Comment in: Circulation. 1996;94(8):1804-6.

11. Patti G, Nusca A, Di Sciascio G. Meta-analysis comparison (nine trials) of outcomes with drug-eluting stents versus bare metal stents in patients with diabetes mellitus. Am J Cardiol. 2008;102(10):1328-34

12. Stettler C, Wandel S, Allemann S, Kastrati A, Morice MC, Schömig A, et al. Outcomes associated with drug-eluting and bare-metal stents: a collaborative network meta-analysis. Lancet. 2007;370(9591):937-48. Comment in: Lancet. 2007;370(9605): 2099-100. Lancet. 2007;370(9591):914-5.

13. Creager MA, Lüscher TF, Cosentino F, Beckman JA. Diabetes and vascular disease: pathophysiology, clinical consequences, and medical therapy: Part I. Circulation. 2003;108(12): 1527-32. Review.

14. Wenaweser P, Daemen J, Zwahlen M, van Domburg R, Jüni $P$, Vaina $S$, et al. Incidence and correlates of drug-eluting stent thrombosis in routine clinical practice. 4-year results from a large 2-institutional cohort study. J Am Coll Cardiol. 2008;52(14):1134-40.

15. lakovou I, Schmidt T, Bonizzoni E, Ge L, Sangiorgi GM, Stankovic G, et al. Incidence, predictors, and outcome of thrombosis after successful implantation of drug-eluting stents. JAMA. 2005;293(17):2126-30. Comment in: JAMA. 2006; 295(1):36; author reply 36. JAMA. 2005;293(17):2154-6.

16. Kuchulakanti PK, Chu WW, Torguson R, Ohlmann P, Rha SW, Clavijo LC, et al. Correlates and long-term outcomes of angiographically proven stent thrombosis with sirolimus- and paclitaxel-eluting stents. Circulation. 2006;113(8):1108-13. Comment in: Circulation. 2006;114(8):e362; author reply e363.
17. Cutlip DE, Windecker S, Mehran R, Boam A, Cohen DJ, van Es GA, Steg PG, Morel MA, Mauri L, Vranckx P, McFadden E, Lansky A, Hamon M, Krucoff MW, Serruys PW; Academic Research Consortium. Clinical end points in coronary stent trials: a case for standardized definitions. Circulation. 2007, 115(17):2344-51.

18. lijima R, Ndrepepa G, Mehilli J, Markwardt C, Bruskina O, Pache J, et al. Impact of diabetes mellitus on long-term outcomes in the drug-eluting stents era. Am Heart J. 2007; 154(4):688-93.

19. Sarnak MJ, Levey AS, Schoolwerth AC, Coresh J, Culleton B, Hamm LL, McCullough PA, Kasiske BL, Kelepouris E, Klag MJ, Parfrey P, Pfeffer M, Raij L, Spinosa DJ, Wilson PW; American Heart Association Councils on Kidney in Cardiovascular Disease, High Blood Pressure Research, Clinical Cardiology and Epidemiology and Prevention. Kidney diseases as a risk factor for development of cardiovascular disease: a statement from the American Heart Association Councils on Kidney in Cardiovascular Disease, High Blood Pressure Research, Clinical Cardiology, and Epidemiology and Prevention. Circulation. 2003;108(17):2154-69. Review.

20. Anfossi G, Russo I, Trovati M. Resistance to aspirin and thienopyridines in diabetes mellitus and metabolic syndrome. Curr Vasc Pharmacol. 2008;6(4):313-28.

21. Angiolillo DJ, Bernardo E, Ramirez C, Costa MA, Sabaté M, Jimenez-Quevedo $\mathrm{P}$, et al. Insulin therapy is associated with platelet dysfunction in patients with type 2 diabetes mellitus on dual oral antiplatelet treatment. J Am Coll Cardiol. 2006; 48(2):298-304. Comment in: J Am Coll Cardiol. 2007;49(5): 628; author reply 628-9.

22. Park DW, Flaherty JD, Davidson CJ, Yun SC, Lee SW, Kim $\mathrm{YH}$, et al. Prognostic influence of diabetes mellitus on longterm clinical outcomes and stent thrombosis after drug-eluting stent implantation in Asian patients. Am J Cardiol. 2009; 103(5):646-52.

23. Mahmud E, Bromberg-Marin G, Palakodeti V, Ang L, Creanga D, Demaria AN. Clinical efficacy of drug-eluting stents in diabetic patients: a meta-analysis. J Am Coll Cardiol. 2008; 51(25):2385-95.

24. Cutlip DE, Chauhan MS, Baim DS, Ho KK, Popma JJ, Carrozza $\mathrm{JP}$, et al. Clinical restenosis after coronary stenting: perspectives from multicenter clinical trials. J Am Coll Cardiol. 2002; 40(12):2082-9

25. Mercado N, Boersma E, Wijns W, Gersh BJ, Morillo CA, de Valk $\mathrm{V}$, et al. Clinical and quantitative angiographic predictors of coronary restenosis: a comparative analisys from the balloonto-stent era. J Am Coll Cardiol. 2001;38(3):645-52.

26. Kastrati A, Dibra A, Mehilli J, Mayer S, Pinieck S, Pache J, et al. Predictive factors of restenosis after coronary implantation of sirolimus- or paclitaxel-eluting stents. Circulation. 2006; 113(19):2293-300. Comment in: Circulation. 2006;113(19): 2262-5.

27. Hong SJ, Kim MH, Ahn TH, Ahn YK, Bae JH, Shim WJ, et al. Multiple predictors of coronary restenosis after drugeluting stent implantation in patients with diabetes. Heart. 2006;92(8):1119-24. 